\title{
LA DERIVACIÓN APRECIATIVA EN LA 23. a EDICIÓN DEL DICCIONARIO DE LA REAL ACADEMIA ESPAÑOLA
}

\author{
MARIA IANNOTTI \\ UNED \\ maria.iannotti@gmail.com
}

\section{RESUMEN}

Una característica del español es la posibilidad de acompañar sustantivos, adjetivos y verbos con una serie de sufijos apreciativos que alteran semánticamente una palabra base, otorgándole una valoración afectiva (positiva o negativa) o atribuyéndole significados connotativos y pragmáticos diferentes dependiendo de la situación comunicativa.

Este trabajo analiza el tratamiento de los sufijos apreciativos en la 23. ${ }^{a}$ edición del Diccionario de la Lengua Española (DLE), así como la información morfológica y etimológica de los apreciativos lematizados que han dado lugar a lexicalización, es decir, a la producción de nuevos términos que han pasado a indicar ciertos referentes y conceptos.

PALABRAS ClAVE: derivación; flexión; lexicalización; morfología; etimología.

\section{RiASSUNTO}

Una caratteristica dello spagnolo è la possibilità di accompagnare sostantivi, aggettivi e verbi con una serie di suffissi alterativi che modificano semanticamente una parola base conferendole un valore affettivo (positivo o negativo) o attribuendole significati connotativi e pragmatici diversi a seconda della situazione comunicativa.

Questo lavoro analizza sia il trattamento dei suffissi alterativi nella $23^{\text {a }}$ edizione del Diccionario de la Lengua Española (DLE), sia l'informazione morfologica ed etimologica degli alterati lemmatizzati che hanno dato luogo a lessicalizzazioni, ossia alla produzione di nuovi termini che indicano determinati referenti e concetti.

PARole chiave: derivazione; flessione; lessicalizzazione; morfologia; etimologia. 


\section{INTRODUCCIÓN}

$\mathrm{La}$ afectividad desempeña un papel importante en el uso de algunos derivados. Por eso resulta significativa la distinción que propone Coseriu (1970: 104-121) entre Bedeutung ('significado') y Bezeichnung ('designación'): el primero se realiza en la lengua (langue) y mantiene la misma unidad funcional; la segunda, en el discurso (Rede) y considera las varias determinaciones connotativas y pragmáticas que este significado asume en los diferentes contextos.

La derivación es un proceso morfológico y léxico por el que un afijo modifica una palabra sin que esta sufra necesariamente cambios en sus propiedades denotativas esenciales ni en su categoría gramatical (aunque pensemos en la existencia de adjetivos denominales, sustantivos deverbales, verbos denominales y también en posibles cambios de género). La diferenciación entre los apreciativos es solo de carácter dimensional y nocional: varía de un sentido cuantitativo, identificable con diminutivos y aumentativos, a otro cualitativo, reconocible por la forma peyorativa. Esta variedad de sentidos es típicamente evaluativa y no parece relacionada con un específico morfema afijal, sino que es propia de todos los procedimientos apreciativos en cuanto tales, aunque es cierto que algunos de derivación resultan más productivos que otros. La derivación apreciativa expresa también valoraciones afectivas y matiza significados; hay algunos derivados que han adquirido una autonomía semántica propia dando lugar a una lexicalización, es decir, a la producción de nuevos términos que, aunque tengan la forma de un derivado apreciativo, han dejado de serlo y han pasado a indicar ciertos referentes o conceptos.

En el presente trabajo se analizarán, por un lado, los rasgos principales de la derivación apreciativa y de su inclusión (o exclusión) en el Diccionario; por otro, se aludirá a la información etimológica de la morfología derivativa tanto de los morfemas como de los lexemas:

Cuando el uso ha dado nuevos valores semánticos a determinados diminutivos, aumentativos y despectivos, el carácter original de tales se indica, por regla general, en la información etimológica [...]. Los diminutivos, aumentativos y despectivos no lexicalizados no tienen por qué incluirse en la nomenclatura del Diccionario. Pero sí constan en él los que implican cambio de género o alguna anomalía derivativa (DLE, 2014: LI).

\section{LA MORFOLOGÍA APRECIATIVA}

La sufijación apreciativa se aplica a los sustantivos, adjetivos, verbos o adverbios. Todo tipo de reparticiones en clases ha de considerarse aproximativo, ya que cada sufijo puede transmitir más de un valor apreciativo a la vez y más de un significado: valoración de tamaño, estima, cortesía, ironía, menosprecio, afectividad, etc. (Lázaro Mora, 1999: 4647-4648). De hecho, ciertos sustantivos y adjetivos admiten varios sufijos, con lo que se obtienen series de palabras similares morfológicamente, aunque distinguibles en sus connotaciones y significado. Así, a partir de libro se forman librillo, librete, libreta, librejo, etc. No obstante, hay que subrayar que no todos los lexemas formados por un afijo valorativo son verdaderos apreciativos. Se pueden encontrar también formas que, en el tiempo, han desarrollado un significado propio, independiente del de la palabra base: 
bastoncillo. (Del dim. de bastón). m. 1. Galón estrecho que sirve para guarnecer. \| 2. Biol. Prolongación cilíndrica fotosensible de ciertas células de la retina de los vertebrados, que recibe las impresiones luminosas incoloras. II 3. Esp. Palillo recubierto de algodón en sus extremos, que tiene diversos usos, especialmente en la higiene personal.

Hay palabras que casualmente terminan con un elemento que puede parecer un sufijo apreciativo, pero que no lo son ni tienen con él una relación semántica originaria. Es el caso de mejilla/mejillón (falso aumentativo), coche/cochino (falso diminutivo), bella/bellota (falso despectivo). Hay veces en que el sufijo apreciativo deja de ser empleado «como expresión libre del punto de vista del hablante y se convierte en un sufijo significativo, que aporta un elemento de significado por el cual una palabra se hace diferente de otra» (Seco, 1989: 209): silla-sillón, camisa-camisón, caja-cajón, etc. En algunos de estos casos la idea de tamaño está presente, pero no es el único rasgo que se diferencia con el objeto designado por la palabra primitiva.

No todas las palabras pueden llevar sufijos: los adjetivos de relación, los ordinales, los sustantivos que denotan características, cualidades y estados físicos o anímicos, por razones categoriales, fonéticas y semánticas, rechazan el uso de los sufijos apreciativos.

Los sufijos apreciativos se ubican a continuación de los morfemas derivativos y antes de los flexivos: come $+d o r+c$-it $+o$ (base + derivación + apreciativo + flexión).

Generalmente, al tratar la derivación apreciativa se suelen considerar solo los fenómenos que atañen a sustantivos y adjetivos. No obstante, hay que integrar también los afijos que se adjuntan a bases verbales. Estos comportan cambios significativos en el aspecto gramatical del verbo que asume un tono de atenuación o de intensificación de la acción expresa o le da sentido iterativo (dormitar, besuquear).

La morfología apreciativa tiene características intermedias entre flexión y derivación no apreciativa: de hecho, al igual que la primera, no produce cambios en la categoría gramatical de la base, pero, como la segunda, afecta al significado inherente de la palabra (Pena, 1999: 4329).

Hay diferentes teorías que tratan de explicar ambos procesos morfológicos: una posición establece una dicotomía entre ellos (Anderson, 1992), con lo que la flexión se referiría al proceso por el cual se crean «nuevas formas de una palabra» y la derivación a la formación de «palabras nuevas». Desde una perspectiva tipológica, hay un conjunto de rasgos que diferencian la flexión de la derivación y, aunque la mayor parte de las propiedades de los apreciativos son derivativas, hay también características flexivas que se distinguen. La morfología apreciativa actúa de manera diferente con respecto a la una y a la otra: a diferencia de la derivación apreciativa, que es opcional y sirve solo para matizar las palabras (aunque eso no impide que muchos apreciativos, por un uso reiterado, lleguen a lexicalizarse), la flexión es obligatoria, ya que es inherente a los procesos de concordancia de la sintaxis; contrariamente a la apreciación, que solo agrega significados connotativos, la derivación forma nuevas palabras. Sin embargo, se pueden encontrar casos ambiguos, con lo cual resulta difícil establecer un límite categórico entre ellas.

La mayoría de los autores considera el sufijo -ísimo un afijo apreciativo derivativo, al igual que el diminutivo y el aumentativo. Esta teoría es contraria a la de la Academia, que 
quiere que se considere morfema flexivo, por faltarle, a diferencia de los derivativos, el valor léxico. Lo cierto es que este morfema incumple también otros criterios de los morfemas derivativos, porque no modifica la categoría de la base (aunque otros sufijos tampoco cambian la categoría de la base: trigo > trigal) y carece de poder generativo, es decir, no crea nuevas palabras. Sin embargo, comparte con los restantes sufijos su incapacidad para constituir cierre de palabra, la inaccesibilidad de la sintaxis o la imposibilidad de funcionar como morfema sincrético. En resumen, -ísimo representaría un elemento de transición entre la flexión y la derivación (García-Page Sánchez, 2011: 152-153).

\section{LA INCLUSIÓN DE LOS DERIVADOS APRECIATIVOS EN EL DLE}

Un asunto muy discutido en el debate metalexicográfico atañe a la inclusión de la sufijación apreciativa en los diccionarios generales. Es cierto que, al ser elementos cargados de significado, podrían considerarse parte integrante del léxico y habría que incluirlos en los textos lexicográficos (Bosque, 1983: 134); no obstante, hay algunos que los relacionan exclusivamente con el ámbito de la gramática por su naturaleza de morfemas ligados (raíz + morfema de género + morfema de número: gat-it-o-s).

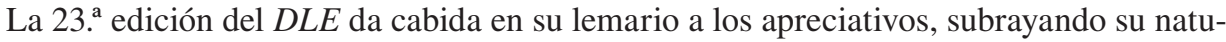
raleza de morfema léxico. Las palabras que contienen derivación apreciativa se dividen en dos grupos: las transparentes y las opacas (no transparentes o lexicalizadas). Las primeras se forman a partir de la combinación de la base y el sufijo, se deduce su significado o matiz de la palabra base, se forman a través de un procedimiento productivo (a excepción de los adverbios diminutivos que el $D L E$ incluye) y, por eso, no suelen aparecer en el diccionario; las opacas, al contrario, se incluyen porque no se obtienen mediante un recurso morfológico activo en la lengua española, sino que ya forman parte del repertorio léxico del idioma (Real Academia Española, 2009: 635-636).

Hay voces que pueden parecer transparentes porque tienen una terminación típica de los sufijos apreciativos, pero que están lexicalizadas porque se han desarrollado de manera diferente con respecto al origen y no se generan a partir de un recurso morfológico añadiendo el sufijo a la base: es el caso de manzanilla, que no se refiere a la «manzana pequeña», sino a la planta herbácea, aunque sea un derivado de manzana (DECH, 1984-1991: tomo III, 830-831).

Los sufijos apreciativos lematizados que se incluyen en la última edición del Diccionario académico son $74^{1}$. Aparecen introducidos por un guion, a fin de señalar su naturaleza de morfemas ligados, y tienen flexión de género (-illo, lla), tal como los sustantivos o adjetivos lematizados.

Tradicionalmente se dividen los sufijos apreciativos dependiendo de la especialización significativa de cada uno de ellos: sufijos aumentativos, diminutivos y peyorativos. Los significados aumentativo y diminutivo no excluyen la existencia de contenidos connotativos afectivos (connotación positiva o negativa), connotaciones que se derivan de la propia

\footnotetext{
${ }^{1}$ Es preciso señalar que algunos remiten a otros.
} 
interpretación que se puede realizar de los significados aumentativo y diminutivo (Rifón Sánchez, 1998: 222):

Il carattere dimensionale e quello nozionale non si escludono, anzi si richiamano a vicenda: alla piccolezza si riferisce la delicatezza e la gentilezza oppure la debolezza e la meschinità; alla grandeza si referisce la forza oppure la cattivería e la brutteza. (Dardano, 1978: 96).

A continuación, se enumeran los sufijos apreciativos lematizados repartidos en las tres clases: aumentativos, diminutivos y despectivos.

Aumentativos:

-al; -aza; -azo, za; -ejón; -icón, na; -ijón, na; -ón1, na; -ote, ta; -udo, da.

Diminutivos:

-achuelo, la; -cete, ta; -cico, ca; -cillo, lla; -cito, ta; -ececico, ca; -ececillo, lla; -ececito, ta; -еcezuelo, la; -ecico, ca; -ecillo, lla; -ecito, ta; -eja; -ejo, ja; -eta; -ete, ta; -eto; -еzno, na; ezuelo, la; -huelo, la; -ichuelo, la; -ina; -ín, na; -ica; - ico, ca; -illa; -illo, lla; -ita3; -ito3, ta; -uelo, la; -zuelo, la.

Despectivos:

-aco2, ca; -aco, ca; -acha; -acho, cha; -aja; -ajo, ja; -alla; -anga; -ango, ga; -aracho, cha; -arda; -ardo, da; -arro, rra; -arraco, ca; -arrajo; -asco, ca; - astra; -astre; -astro, tra; -esco, ca; -inga; -ingo, ga; -ijo; -isco, ca; -izco, ca; -orrio, rria; -orro, rra; -rro, rra; sco, ca; -strajo; -ucho, cha; -uco, ca; -usco, ca; -uzco, ca.

Es frecuente incluir los aumentativos y los despectivos en el mismo grupo, dado que tienen algunos rasgos en común. De hecho, cuando el aumentativo tiene que ver con el tamaño, se acerca a los despectivos, ya que aporta a la base la idea «de grande o excesivo, habitualmente unida a la expresión de escasa simpatía — repulsa o burla— por parte del hablante hacia lo que menciona» (Seco, 1989: 208):

cabezota. adj. 1. coloq. Que tiene la cabeza grande. U.t.c.s. II 2. coloq. Terco, obstinado. U.t.c.s.

Entre los sufijos aumentativos, los que terminan en -ón, -azo generalmente indican tamaño excesivo (platón, golpetazo); otros añaden la idea de afecto, -ote, -ota (niñote) que, junto al valor aumentativo, tienen el de despectivo (grandote). Bajo el lema -ote, ta el DLE sugiere las palabras bobote, grandote, angelote, marquesota, aumentativos de adjetivos y nombres con matiz despectivo. Angelote y marquesota ${ }^{2}$ se encuentran también lematizadas en el Diccionario ${ }^{3}$.

${ }^{2}$ El étimo del término marquesota es marquesote ( «torta de forma de rombo, hecha de harina de arroz o de maíz, con huevo, azúcar, etc., y cocida al horno»), que, a su vez, es un lema lexicalizado que procede del aumentativo despectivo desusado marqués. Así pues, este último ha dado vida a dos palabras polisémicas en las que los diferentes significados se relacionan etimológica y semánticamente.

3 angelote. m. 1. de talla o pintura de un ángel niño gordezuelo. II 2. afect. coloq. Niño especialmente de corta edad. I| 3. Persona bondadosa, especialmente de gran tamaño y proporciones. || 4. Pez selacio del suborden de los escuá- 
El sufijo de origen latino -ón (y los que se combinan con él, -acho, -ajo, -arro, -ejo, -ete) conserva su significado de tamaño desmesurado o un defecto sobre todo referido a partes del cuerpo, produciendo una recategorización, dado que la forma primitiva era sustantiva y, al agregarse, el aumentativo en muchos casos se ha convertido en adjetivo (cabeza/cabezón).

Muchos de los adjetivos en -ón, na denotan crítica amable, ironía, censura: tristón, por ejemplo; resulta despectivo solterón/solterona. En el artículo -ón, na se incluye también la información etimológica: (Del lat. -o, -ōnis). Generalmente, cuando el adjetivo del que procede el aumentativo no tiene valoraciones particulares, el derivado que se obtiene denota intensificación y, a veces, abundancia o exceso (dulzón equivale a «demasiado dulce»). Hay muchas palabras con el sufijo -ón que ya están lexicalizadas y tienen un significado distinto del de la base: cinturón, salón, etc.

En ocasiones, el aumentativo -ón y, a veces, también -ote, no aportan ni un significado aumentativo ni despectivo, sino atenuativo, que modera afirmaciones demasiado agresivas por parte del hablante y que pueden llegar a funcionar como un diminutivo: tristón, seriote, etc. El aumentativo comparte los valores semánticos de los sustantivos, adjetivos o adverbios a los que se añaden. En los nombres aporta un significado más referencial, porque se relaciona con el tamaño físico; por el contrario, en los adjetivos tiene un significado más tenue, ya que adopta tanto valores aumentativos como matices atenuativos, incluso usos irónicos o despectivos. Los aumentativos -icón, na e -ijón, na tienen valores entre aumentativo y despectivo (meticón, metijona); el sufijo - udo, -uda indica abundancia, gran tamaño, intensidad con respecto al significado de la raíz (barbudo).

El sufijo - $a z o$, $z a$, en el habla informal, sustituye frecuentemente al superlativo o al adverbio de cantidad muy (buenaza). Se puede aplicar a palabras que indican mucha cantidad (hambrazo, calorazo), no suele formar adjetivos y se usa con valor sustantivo (buenazo). Es frecuente el uso de estos sufijos para indicar una valoración positiva (madraza, regalazo, golazo, partidazo). Otro uso del aumentativo es aquel que imprime a los sustantivos y a los adjetivos un sentido de acción rápida, momentánea, relacionada con los objetos a los que se aplica (telefonaza, enamoradazo). Para aquellos adjetivos que indican un estado más duradero, se prefiere el sufijo -ón, na (solterón). Muchos de ellos son ponderativos o aumentativos (peliculón) y, solo a veces, despectivos, dependiendo del contexto. El significado tradicional del aumentativo -azo se relaciona con 'golpe' o 'acción ejercida con determinado instrumento o determinada parte del cuerpo’ (portazo). Antes, el sufijo con el valor de golpe (o con el de acción brusca) era -ón, y dio lugar a formaciones como apretón, pisotón. Sin embargo, en estos casos este sufijo estaba asociado más al cuerpo que a golpes ejecutados con objetos, es decir, la base se relacionaba con la parte del cuerpo que recibía la acción. Por fin, el sufijo - $u d o$, da indica abundancia, gran tamaño, intensidad con respecto al significado de la raíz (barbudo).

Es cierto que estas expresiones se acompañan también de una entonación para evidenciar el carácter enfático de las palabras:

\footnotetext{
lidos, que llega a tener dos metros de largo, aplastado, de color azul oscuro por encima y blanco por debajo, de cabeza redonda y con aletas pectorales y abdominales muy grandes, a manera de alas blancas. II 5. Especie de higueruela.

marquesota. (De marquesote). f. Cuello alto de tela blanca que, muy almidonado y hueco, usaban los hombres como prenda de adorno. - a la . loc. adj. desus. Dicho de un vestido: De una hechura especial.
} 
«un poderoso vehículo de la connotación afectiva; hasta el punto de que es capaz de colorear cariñosamente a términos denotativamente ofensivos» (Lázaro Mora, 1999: 4647).

Las voces despectivas se forman también con otros sufijos apreciativos, -aco, ca: libraco (cuando se combina con -arro, toma la forma -arraco con valor diminutivo y despectivo: pajarraco); -acho, cha: poblacho (con el sufijo -ar toma la forma -aracho, vivaracha); -astro, tra: poetastro, madrastra (aunque este último término ha perdido el valor despectivo y, actualmente, indica solo 'la mujer del padre de una persona nacida de una unión anterior de este'), como variante, -astre, pillastre; -ato, ta: niñato; tanto -orrio, rria como -orro, rra envían a -rro, rra (vidorra, bodorrio, ventorro). Finalmente, el sufijo -ajo, ja que forma sustantivos y adjetivos con valor entre despectivo y diminutivo: tendajo, migaja, escobajo, pequeñajo. Puede combinarse con -ar: espumarajo. También se combina con -arro: pintarrajo. A veces toma la forma -strajo: comistrajo. Estas combinaciones tienen valor despectivo. De las siete palabras incluidas como ejemplo en el $D L E$ solo una es propiamente un diminutivo con matiz afectivo o despectivo, pequeñajo, mientras que las demás son derivados lexicalizados. Específicamente, en las voces escobajo, pintarrajo y comistrajo no aparece la relación derivacional con la base ni referencia alguna a su derivación apreciativa (aunque se deduce que escobajo procede de escoba, pintarrajo de pintura, comistrajo de conmisto); en tendajo, migaja y espumarajo, al contrario, está presente la información etimológica y se advierte que proceden respectivamente del despectivo de tienda, del diminutivo de miga y del despectivo de espuma.

Los diminutivos no cambian el significado de las palabras de las que proceden, pero aminoran el tamaño y contribuyen a reforzar la expresividad y a enfatizar lo que se dice, indicando una disminución de tipo cuantitativo y cualitativo. Por su misma naturaleza son de libre elección por parte del hablante y su función semántica va dirigida a expresar la afectividad más que el tamaño, es decir, la función emotiva del lenguaje prevalece sobre la referencial de indicación de un tamaño pequeño. El matiz afectivo (pequeñin, majete) se alterna con el valor atenuativo (debilucho), cariñoso (chiquilla) y hasta despectivo (casucha, libraco). Estos sufijos se utilizan también en funciones comunicativas específicas para una forma de cortesía (gordito), para atenuar una orden o una solicitud (calladito), ironizar ( Menuda casita!). Hay palabras que con el tiempo han perdido la connotación diminutiva del origen y se han lexicalizado (pañuelo, ganchillo).

En español, el diminutivo aparece mayoritariamente en la variante morfológica -ito, $t a$, que, en ciertos casos, toma las formas -cito, ta; -ecito, ta; -ececito, ta y que, junto a los sufijos -ico, ca; -ín, na, generalmente, tiene valor afectivo (hermanito). Se añade a cualquier categoría gramatical, bien sustantivos (camita), bien adjetivos (gordito) o adverbios (cerquita) y también a verbos (en la forma de gerundio, caminandito, aunque se documentan pocos ejemplos y sobre todo en el estrato culto) y a nombres propios (Alvarito) y no resulta normal emplearlo dentro de la combinatoria de sufijos. El valor afectivo del sufijo -ito, ta es indudable y está relacionado con la expresión de 'menor que'; es el diminutivo más empleado, ya que resulta el comodín para muchas fórmulas de expresión y de afecto. Por esta razón es probablemente el que aporta más significaciones y matices al término conceptual al que se refiere: cariñoso, halagador, insistente y superlativo e irónico y despectivo. No obstante, es indiscutible el predominio del diminutivo afectivo sobre el empleo etimológico del morfema de diminutivo que, procedentes del latín vulgar îttus, se refiere al tamaño e indica pequeñez. 
Destacan también por su estilo los diminutivos reforzados, es decir, los que aparecen con una reiteración del vocablo (gordito, gordito), repetición del morfema (chiquitito) o por adición de una locución adverbial (poquillo gordito). A veces, tanto en su valor halagador como en el irónico despectivo el diminutivo puede ir precedido por una partícula exclamativa que refuerza el conjunto del enunciado (menudo, qué, vaya, etc.).

El matiz de insistencia o superlativo se consigue de dos maneras: añadiendo el sufijo -ito a adverbios (rapidito), locuciones adverbiales o formas verbales no personales; o repitiendo el adjetivo o adverbio al que se ha añadido el sufijo -ito (despacito despacito). El hecho de reiterar los términos (sobre todo adjetivos, adverbios y formas verbales no personales) contribuye a subrayar insistencia y superlación (rapidito equivale a muy rápido), es decir, su empleo hace que de algún modo se subraye el significado de la base lexemática a la que acompaña, siendo por tanto la base la que ofrece un valor de diccionario o valor léxico, y el diminutivo, el que añade un valor de insistencia (Alonso, 1967: 165).

El matiz irónico y despectivo se realiza cuando el sufijo -ito se añade a sustantivos y (menos) a adjetivos, aplicando la entonación correspondiente: ¿El señorito no quiere hacer los deberes?

Tanto el sufijo femenino -ica como las formas -ico, ca se incluyen en el DLE. El primero denota valor iterativo y despectivo, aun teniendo también un significado diminutivo o afectivo, tal como aparece en el sufijo -ico, ca. Werner (1982: 297-328), hablando de homonimia y polisemia, afirma que, si el origen de un sufijo es común, habría que añadir la acepción de significado diminutivo al lema -ica; si no es así, hay que distinguir entre -ica ${ }^{1} e-i c a^{2}$, donde uno de los dos remite al lema flexionado coherentemente con los otros sufijos.

Lo mismo se puede aplicar al sufijo -ina, dado que incluye entre sus varias acepciones el valor apreciativo. Tampoco en este caso el $D L E$ señala el vínculo con el lema flexionado -ín, na. No aparece la variante masculina -ino, que Felíu Arquiola (2009: 64) señala como -ín(o), na. Este sufijo lo encontramos con sustantivos masculinos de diferentes significados. Muy a menudo aparece lexicalizado: maletín, sillín.

El sufijo -illo es uno de los más prolíficos en español. Tiene un significado diminutivo y afectivo y se aplica a sustantivos (pajarillo), adjetivos (flaquillo), adverbios (despacillo), formas verbales no personales (corriendillo) y nombres propios (Pepillo). Con sus variantes, -cillo, -ecillo, y los femeninos, tiene valor diminutivo y afectivo y se comporta exactamente igual que el sufijo -ito, ta, tanto que se pueden sustituir por este sufijo: pajarito, flaquito, despacito, corriendito, Pepito. Es frecuente que -illo se use más con lexicalizaciones, mientras que -ito exprese propiamente la pequeñez: bolso -bolsito -bolsillo. Sin embargo, hay casos en que -ito también se encuentra con lexicalizaciones: cabrito se refiere a la cría de la cabra.

Juan de Miranda sostiene que «la diferencia que hay entre -ito e -ico, por un lado, e -illo es que mientras en -ito e -ico siempre se advierte un modo de afecto, en -illo sólo hay la idea de disminuir alguna cosa sin otra consideración, ni de amor, ni de afecto» (apud Lázaro Mora, 1999: 4648). A veces, -illo puede evidenciar un matiz peyorativo (en contraste con -ito), acercándose más al despectivo en -ejo (animalillo, animalejo). 
Se puede notar que en el $D L E$ hay algunos sufijos lematizados en la forma femenina y otras no. Es el caso de -illa, pero no de -ucha, que solo se encuentra flexionada en masculino y femenino (-ucho, cha). Puede observarse que casi siempre los sufijos que no presentan las formas lematizadas del femenino son el resultado de la combinación de un interfijo interradical y un sufijo (Felíu Arquiola, 2009: 66), excepto en dos casos: por un lado, falta un lema femenino lematizado aparte para -ón, na, -ote, ta y -исо, $c a$, sufijos apreciativos sin interfijos; por el otro, aun combinando el interfijo -ar- y el sufijo -acho, cha, -aracha aparece lematizado y remite al lema flexionado -aracho, cha, que a su vez envía a -acho, cha. Se observa también que se incluyen lematizadas, con remisión al sufijo de origen, las variantes de formas bien con adición de interfijos (-ececito, ta. V. -ito ; - ecito, ta. V. ito ${ }^{3}$; - cito ${ }^{2}$, ta. V. -ito ${ }^{3}$ ) bien con combinación con otros sufijos (-achón. V. -ón'; -ajón. V. ón'i astro, na. V. -ón').

El DLE remite la entrada correspondiente al lema -illa a -illo, es decir, a un lema en el que se explicita exclusivamente la forma masculina, aunque se encontrará el lema flexionado -illo, lla. Muy probablemente, de acuerdo con el proceso de homologación de la lematización de los sufijos apreciativos, todas las formas femeninas lematizadas desaparecen y se encuentran flexionales en un único artículo. Por esto desaparecen (respecto a la edición anterior de 2001) también los lemas -aca, -acha, -aja, -aracha, -arda, -astra, -aza, -eja, -eta, -ita, que antes remitían al respectivo lema masculino. Solo en poquísimos casos todavía aparece aparte con remisión al lema masculino: -acha, V. -acho; -astra. V. astro; -ata. V. -ato'; -aza. V. -azo; - eja. V. -ejo; -ela. V. -elo; -enga. V. -engo; -eta. V. -ete; -illa. V. -illo; -inga. V. -ingo; -ita ${ }^{3}$ V. -ito

A los sufijos -uelo, -uela (a veces toman las formas -ecezuelo, -ezuelo; -zuelo; -achuelo e -ichuelo; -huelo) se les considera diminutivos y despectivos, dependiendo del contexto en que se utilizan. En algunos casos han perdido su significado diminutivo y se han lexicalizado: pañuelo. De hecho, tras lexicalizarse, -uelo, la ha perdido todo su valor diminutivo.

Este es un sufijo muy productivo en cuanto a su valor afectivo (hoyuelo, pequeñuelo), pero es menos frecuente que los anteriores.

Por fin, con -ete, ta se forman diminutivos, despectivos o, incluso, palabras de valor afectivo; suelen incluir rasgos humorísticos, atenuación, complicidad: mentirosete, amiguete, calvete. Puede tener usos exclusivamente estilísticos, pero no es de los más frecuentes.

\section{LOS DERIVADOS APRECIATIVOS LEMATIZADOS}

Los apreciativos lexicalizados son palabras que presentan los sufijos típicos de la derivación, pero tienen un significado propio, completamente autónomo y diferente del de un derivado, es decir, la originaria relación apreciativa entre las palabras se ha perdido (el bastoncillo, como ya se ha visto, no es el «bastón pequeño», sino, entre otras cosas, un "galón estrecho»), transformándose en una relación de derivación, que muy a menudo no es tan patente para todos los hablantes, pero que es posible reconstruir por vía etimológica. En el $D L E$ hay muchas palabras lexicalizadas. A continuación se presentan algunas de ellas, que proceden de una derivación apreciativa y se analiza la variación del tratamiento de la información sobre el proceso derivacional que la palabra ha sufrido originariamente. 
presilla. (Del dim. de presa). f. 1. Cordón o tira pequeña de tela que se pone en una prenda para enganchar un botón o un corchete o para sujetar un cinturón. II 2. Anilla o pieza que sirve para enganchar o sujetar algo. ॥ 3. Am. Distintivo de ciertos uniformes para indicar graduación. ॥ 4. Cuba. grapa (II pieza metálica para sujetar papeles). II 5. p. us. Punto de costura que se utiliza para reforzar los bordes de una tela. $\| \mathbf{6}$. desus. Tela basta de lino.

Generalmente, en las lexicalizaciones se incluye o la información morfológica de la palabra o su etimología. En el caso de la palabra presilla, el $D L E$ señala entre paréntesis la información derivacional, la base nominal, presa, y su proceso apreciativo que indica una derivación con valor diminutivo (Del dim.).

En el caso de la palabra sillón, se hace referencia al proceso derivacional apreciativo aportado por el sufijo -ón de manera indirecta en las dos acepciones del artículo lexicográfico:

sillón. m. 1. Silla de brazos, mayor y más cómoda que la ordinaria. I| 2. Silla de montar construida de modo que una mujer pueda ir sentada en ella como en una silla común. $\square \sim$ de hamaca. m. Ur. mecedora.

Los dos ejemplos son significativos porque tienen una información diferente: el primero indica la procedencia y la valoración apreciativa; el segundo las deduce de las acepciones. En parte, esto se justifica por el hecho de que la palabra presilla ha dado nuevos valores semánticos a la palabra de la que procede, es decir, transmite un significado no fácilmente deducible de su base; en cambio, el significado de sillón es mucho más parecido al de su origen.

A continuación, analizamos la palabra manitas para aclarar el proceso de lexicalización que implica un cambio notable en el significado y para distinguir una palabra lexicalizada de otra con valoración apreciativa.

Manitas es semánticamente ambigua, porque tiene significados diferentes: "persona que tiene gran habilidad para una actividad manual o un oficio» $\mathrm{y}$ "manos pequeñas» (García-Page Sánchez, 2011: 65). Esta diferencia de significados se refleja en la estructura de la palabra: [[man-]-itas] y [[[man-]-ita]-s]. Se puede observar que la estructura morfológica es diferente aunque el origen sea el mismo: la primera se ha lexicalizado con un significado distinto (aunque, sin duda, relacionado) al del origen; la segunda tiene un valor apreciativo diminutivo de mano. Resumimos el proceso morfológico de esta manera: base $($ mano $)>$ derivado apreciativo (manita) $>$ lexicalización del derivado apreciativo (manitas). El reenvío al sustantivo de base tiene un valor etimológico y semántico al mismo tiempo, dado que indica un proceso de derivación además de mostrar la naturaleza apreciativa de la voz.

La estructura de las voces dedicadas a los derivados apreciativos verbales es análoga a la de todas las otras. En su etimología se indica la derivación desde el verbo de base, aunque no se especifique el sufijo valorativo. El DLE no parece comportarse coherentemente al incluir la información derivacional de los apreciativos verbales. De hecho, a veces aparece la información morfológica; otras veces, la procedencia de una base ya apreciativa; y en algunos casos no se incluye ninguna de las dos:

pintorrear. (De pintar). tr. coloq. pintarrajear (II pintar sin arte). 
besuquear. (De besucar). tr. coloq. Besar repetidamente a alguien o algo.

bailotear. int. coloq. Bailar mucho, y en especial cuando se hace sin gracia ni formalidad.

Analizando la estructura de estos verbos es posible evidenciar que, generalmente, se suele formar derivados verbales (en -ear, sobre todo) añadiendo interfijos y, por eso, la interfijación se produce generalmente junto a la sufijación: [pint-[-orr- + -ear]]; [bes-[-uqu- + -ear]]; [bail-[-ot- + -ear]]. No obstante, hay derivados verbales en los que a la base léxica se añade directamente el sufijo: [color-[-ear]].

Los verbos apreciativos pertenecen todos a la primera conjugación, independientemente de la conjugación del verbo de la base, porque el sufijo que se emplea es -ear. El sufijo puede producir en el verbo un significado atenuativo (beborrotear 'beber a menudo y en poca cantidad'), intensivo (traquetear 'hacer ruido, estruendo o estrépito), despectivo (lloriquear 'llorar sin fuerza y sin bastante causa') o modifica el aspecto verbal indicando en la mayor parte de los casos una acción reiterada (besuquear 'besar repetidamente a alguien o algo').

A veces, aparece un sufijo verbal en el derivado inexistente en la base (picar > picotear) o, al revés, desaparece un sufijo existente (carrear > carrejar), por el truncamiento de la marca verbal de la base, ya sea esta vocal temática, ya sea sufijo verbal (Rifón Sánchez, 1998: 210). En realidad, por lo expuesto anteriormente, si situamos la aplicación de las reglas apreciativas entre el fenómeno de la derivación y el de la flexión, los afijos apreciativos tendrían que ser sufijos que se unen a un tema (derivado o no) que sirve como base para crear otro tema.

No se justifica en el $D L E$ la evolución de algunas palabras que remiten a una derivación desde una base nominal hasta el verbo apreciativo, sin indicar en la etimología la relación entre la base nominal y el verbo:

traquear. (De traque). intr. p.us. traquetear.

traquetear. (De traque). tr. 1. tr. Mover o agitar algo de una parte a otra. Se usa especialmente hablando de los líquidos. II 2. coloq. Frecuentar, manejar mucho algo. o intr. Hacer ruido, estruendo o estrépito.

El análisis del verbo apreciativo traquetear podría resultar más plausible si la información etimológica indicase traquear como base de formación del verbo al que se añade el interfijo -et- y el sufijo verbal -ear, según una estructura morfológica [[traqu-[-et-]-ear]. Considerando que la clase de morfemas que intervienen son respectivamente el lexema, el interfijo y el sufijo verbal, y que, como ya se ha dicho, la interfijación y la sufijación se producen conjuntamente, se realizaría el siguiente proceso de formación: traqu- (lexema) + -et- (interfijo) + -ear (sufijo verbal), determinando una evolución del verbo traquetear a partir de una base nominal, traque $>$ traquear $>$ traquetear.

\section{CONCLUSIONES}

Una de las características del español es el frecuente uso de morfemas apreciativos que se añaden a sustantivos, adjetivos y, ocasionalmente, a verbos, para expresar tamaño, atenuación, encarecimiento, cercanía, ponderación, cortesía, ironía, menosprecio y otras nociones 
que caracterizan la valoración afectiva que se hace de las personas, los animales o las cosas (Real Academia Española, 2009: 627).

El análisis de la derivación apreciativa pone de relieve la diferente naturaleza que muestran los morfemas flexivos y los derivativos: un proceso sintáctico el primero, un proceso léxico el segundo, aun teniendo características comunes que colocan a la morfología apreciativa en una posición intermedia entre la flexión y la derivación.

Analizando los afijos apreciativos lematizados y, asimismo, las formaciones apreciativas lexicalizadas incluidas en la 23. ${ }^{a}$ edición del Diccionario de la Real Academia Española, se ha podido constatar que no siempre se tratan de manera coherente y homogénea y que la información morfológica o etimológica que aparece en el paréntesis después del lema muy a menudo solo ofrece la base a la que se añaden los elementos apreciativos y muy raramente proporciona una información detallada de los elementos que participan en el proceso morfológico.

\section{REFERENCIAS BIBLIOGRÁFICAS}

Anderson, Stephen R. (1992): A Morphous Morphology, Cambridge: Cambridge University Press.

Bosque, Ignacio (1983): «La morfología», en Francisco Abad y Antonio García Berrio (Eds.), Introducción a la Lingüística, Madrid: Alhambra.

Corominas, Joan, y Pascual, José Antonio (1984-1991): DECH, Madrid: Gredos.

Coseriu, Eugenio (1970): «Bedeutung und Bezeichnung im Lichte der strukturellen Semantik», en Peter Hartmann y Henri Vernay (Eds.), Sprachwissenschaft und Übersetzen, München: Hueber.

DaRDANo, M. (1978): La formazione delle parole nell'italiano di oggi, Roma: Bulzoni.

Felíu Arquiola, Elena (2009): «Palabras con estructura interna», en Elena de Miguel (Ed.), Panorama de la Lexicología, Madrid: Ariel.

García-Page Sánchez, Mario (2011): Cuestiones de morfología española, Madrid: Editorial Universitaria Ramón Areces.

Lázaro Mora, Fernando A. (1999): «La derivación apreciativa», en Ignacio Bosque y Violeta Demonte (Dirs.), Gramática descriptiva de la lengua española. 3. Entre la oración y el discurso. Morfología, Madrid: Espasa Calpe.

Pena, Jesús (1999): «Partes de la morfología. Las unidades del análisis morfológico», en Ignacio Bosque y Violeta Demonte (Dirs.): Gramática descriptiva de la lengua española, Madrid: Espasa Calpe.

Real Academia Española (2009): Nueva gramática de la lengua española, Madrid: Espasa Libros.

- (2001): Diccionario de la lengua española, 22. ${ }^{a}$ ed., Madrid: Espasa Calpe.

- (2014): Diccionario de la lengua española, 23. ${ }^{a}$ ed., Madrid: Espasa Calpe.

Rifón SÁNCHEZ, A., (1998): La derivación verbal apreciativa en español. ELUA 12.

Seco, Manuel (1989): Gramática esencial del español - Introducción al estudio de la lengua, 2. ${ }^{\text {ed., }}$ Madrid: Espasa Calpe.

WERNER, REINHOLD (1982): «Homonimia y polisemia en el diccionario». En Günther Haensch, Lothar Wolf, Stefan Ettinger y Reinhold Werner (Eds.): La lexicografía. De la lingüística teórica a la lexicografía práctica. Madrid: Gredos. 